\title{
Dispositifs de coordination entre industriel, éleveurs et périmètre irrigué dans un bassin de collecte laitier au Maroc
}

Pierre-Yves Le Gal ${ }^{1}$

Marcel Kuper ${ }^{2}$

Charles-Henri Moulin ${ }^{3}$

Laurence Puillet ${ }^{3}$

Mohamed Taher Sraïri ${ }^{4}$

${ }^{1}$ Centre de coopération internationale

en recherche agronomique

pour le développement (Cirad),

Unité mixte de recherche (UMR)

"Innovation",

TA C-85/15,

34398 Montpellier cedex 5

<pierre-yves.le_gal@cirad.fr>

${ }^{2}$ Centre de coopération internationale en recherche agronomique

pour le développement (Cirad),

Unité mixte de recherche (UMR) " G-EAU »,

Institut agronomique et vétérinaire (IAV)

Hassan II,

BP 6202,

Madinate Al Irfane

10101 Rabat

Maroc

$<$ m.kuper@iav.ac.ma>

\section{${ }^{3}$ SUPAGRO}

Unité mixte de recherche (UMR) ERRC,

2, place Viala, 34060 Montpellier cedex 2,

France

<moulinch@supagro.inra.fr>

<laurencep1@free.fr>

${ }^{4}$ Institut agronomique et vétérinaire (IAV)

Hassan II,

Département productions animales,

BP 6202

Madinate Al Irfane

10101 Rabat

Maroc

$<$ mt.srairi@iav.ac.ma>

\begin{abstract}
Résumé
Le concept de chaîne d'approvisionnement est appliqué à un ensemble composé d'un bassin de collecte laitière adossé à un grand périmètre irrigué marocain pour en comprendre le fonctionnement. Quatre grands types d'acteurs sont identifiés, à savoir : le gestionnaire de périmètre, l'industriel laitier, les coopératives de collecte et les éleveursirrigants. Leurs relations sont analysées autour des dispositifs mis en place pour coordonner leurs décisions par rapport aux objectifs d'économie et de valorisation de l'eau (côté périmètre), de qualité, saisonnalité et productivité (côté bassin de collecte). Ils s'articulent autour de la planification et du pilotage des flux d'eau et de lait, d'outils économiques (système de tarification de l'eau, système de paiement du lait), d'échanges d'informations et de services liés (appui technique, fourniture d'intrants et de crédit). Les performances observées sont cependant perfectibles et différents axes de rechercheintervention sont proposés en conclusion : aide à la conception de système d'alimentation des vaches laitières sous contraintes, gestion de la qualité du lait de l'étable à l'usine et gestion des coopératives.
\end{abstract}

Mots clés : aspect économique ; chaîne d'approvisionnement ; collecte du lait ; Maroc.

Thèmes : productions animales ; systèmes agraires ; économie et développement rural ; eau.

\section{Abstract}

Co-ordination processes between a dairy plant, dairy farms and an irrigation scheme in a Moroccan milk supply area

The supply chain concept is used for analyzing the management of a dairy plant supply area located in a large Moroccan irrigation scheme. Four main stakeholders are identified, i.e. the scheme manager, the dairy company, milk collection co-operatives and dairy farmers using irrigation water. The tools and processes implemented in order to co-ordinate their decisions are analyzed considering objectives such as saving irrigation water and increasing the water productivity on the water supplier side, and improving the milk quality, the seasonality and the productivity as far as the dairy supply chain is concerned. It includes: i) planning and operating both the water and milk flows; ii) implementing adapted economic tools, i.e. water pricing and milk payment system; and iii) providing services to farmers such as technical advice and input/credit supply. Nevertheless, the system performances could be improved. Various research and intervention topics are suggested in the conclusion, mainly: i) the design of a decision support system dedicated to the cows' feeding system; ii) the milk quality management along the supply chain; and iii) the co-operatives' management system.

Key words: economic aspects; milk collection; Morocco; supply chain.

Subjects: animal productions; farming systems; economy and rural development; water.

Tirés à part : P.-Y. Le Gal 
es industries agroalimentaires fournissent des revenus aux exploitations familiales qui les approvisionnent en matières premières. Ces ensembles constitués de clients et fournisseurs autour d'un produit agricole évoluent dans un environnement économique concurrentiel et sont à la recherche de gains d'efficacité pour demeurer rentables. Ces gains sont d'abord recherchés au niveau de chaque opérateur: comment augmenter les rendements et diminuer les coûts de production de la matière première dans les exploitations agricoles? Comment mieux coordonner les fonctions d'approvisionnement, de transformation et de distribution dans l'entreprise agroalimentaire?

Ces questions demeurent pertinentes mais se posent aujourd'hui dans le cadre d'ensembles plus larges, incluant l'ensemble des acteurs appartenant à la même chaîne d'approvisionnement. Cet article montre comment l'organisation et le fonctionnement de ces chaines se traduisent sur un bassin de collecte laitier adossé au périmètre irrigué du Tadla, au Maroc. Après un cadrage théorique, les dispositifs tant logistiques qu'économiques mis en place pour coordonner les actions des différents acteurs impliqués sont analysés, puis des perspectives sont ouvertes en termes d'activités de recherche tournées vers l'action.

\section{Cadre théorique et méthodologique}

Une chaîne d'approvisionnement se définit comme un ensemble de relations coordonnées entre fournisseurs, industriels, distributeurs et commerçants assurant la transformation d'une matière première en des biens de consommation finale (Beamon, 1998). Elle porte à la fois sur la planification et le contrôle des flux et sur les activités logistiques proprement dites au sein de la chaîne (Chen et Paulraj, 2004). Organiser et gérer une chaîne d'approvisionnement renvoient donc aux trois niveaux de décision classiquement rencontrés en gestion de production (Giard, 1988) : opérationnel pour le pilotage des flux sur un horizon infrahebdomadaire, tactique pour la planification des opérations sur un pas de temps plurimensuel, stratégique pour les décisions d'investissement et de dimensionnement des ressources à mobiliser aux différents maillons de la chaîne. Celle-ci devient alors l'unité de gestion privilégiée pour analyser les phénomènes de compétitivité et améliorer l'efficacité des processus productifs (Croom et al., 2000).

Ces questions sont en général traitées sur des cas industriels comprenant des fournisseurs peu nombreux, éventuellement dispersés sur plusieurs pays et disposant d'un bon niveau d'information et de contrôle de leurs process (Shah, 2005). Les industries agroalimentaires de première transformation, telles que le lait ou le sucre, se distinguent de ces situations. D'une part, leur chaîne d'approvisionnement est reliée à un bassin d'approvisionnement bien délimité géographiquement et défini par l'espace comprenant une unité industrielle et l'ensemble des producteurs qui la fournissent en matière première (Le Bail, 2005). D'autre part, l'atomisation des exploitations agricoles et l'hétérogénéité des lots livrés en quantité et qualité, sont sources d'incertitudes et d'aléas dans l'organisation des approvisionnements des unités de transformation (Gaucher et al., 2004). Enfin le caractère périssable de la matière première agricole et la complexité de ses caractéristiques influencent les modes d'évaluation et de suivi des lots.

\section{Un ensemble périmètre - bassin de collecte}

\section{vu comme une seule chaîne d'approvisionnement}

Le bassin de collecte laitier du Tadla, situé à $200 \mathrm{~km}$ au sud-est de Casablanca, se distingue des bassins de collecte laitiers des pays développés basés sur de grandes exploitations, et des situations rencontrées en Afrique de l'Ouest, où des mini-laiteries s'approvisionnent auprès d'éleveurs pastoraux et assurent une transformation minimale de la matière première (Corniaux et al., 2005). Il comprend quatre entités aux fonctions bien différenciées :

- un périmètre irrigué d'environ 100000 hectares, géré par une agence para-étatique, l'Office régional de mise en valeur agricole du Tadla (Ormvat). L'Office assure la distribution de l'eau aux exploitations agricoles via un réseau gravitaire ;
- une laiterie industrielle traitant 114 millions de litres annuellement, provenant pour l'essentiel du périmètre irrigué. Elle injecte ainsi environ 345 millions de dinars $(1$ euro $=11$ dirhams $)$ dans l'économie locale, à $95 \%$ sous la forme de lait brut payé aux éleveurs, complétés des primes de qualité versées aux coopératives de collecte ;

- 17000 exploitations d'élevage, dont les cultures fourragères (luzerne essentiellement à laquelle s'ajoutent 3000 hectares de maïs ensilage et du trèfle d'Alexandrie) occupent $25 \%$ de la surface irriguée du périmètre. Ces exploitations gèrent un cheptel total de 55000 vaches laitières produisant environ 175 millions de litres de lait, dont une part est autoconsommée par les veaux et les familles. L'activité laitière génère une production de viande basée sur la vente des animaux en surplus après engraissement ;

-84 coopératives de collecte laitière jouant un rôle d'intermédiaires entre les éleveurs individuels et la laiterie en gérant des centres de collecte munis de citernes réfrigérées où les éleveurs viennent livrer leur production.

Cette conjonction entre périmètre irrigué et agro-industrie présente de nombreux avantages croisés. Elle offre à l'industriel une relative sécurisation de ses livraisons du fait de la meilleure maîtrise de la production associée à l'irrigation (Sraïri et Khattabi, 2001), tout en réduisant ses coûts logistiques grâce à une concentration spatiale des fournisseurs illustrée par la figure 1. En retour, l'activité laitière est génératrice de revenus agricoles, qui facilitent le paiement des redevances hydrauliques perçues par le périmètre pour la fourniture du service de l'eau aux éleveurs. Les relations entre éleveurs, gestionnaire du périmètre et laiterie s'inscrivent dans une chaîne d'approvisionnement décrite à la figure 2. Cette chaîne part de la fourniture de l'eau par l'Ormvat, à partir d'une ressource de surface elle-même gérée par une agence de bassin. Ce service fait l'objet d'une première fonction de production $F_{p}{ }^{1}$ transformant l'eau brute fournie par l'agence en un ensemble de livraisons auprès des irrigants, dont les exploitations laitières.

La transformation de l'eau en fourrages relève d'une deuxième fonction de production $F_{p}{ }^{2}$ incluant les modes de conduite technique des cultures fourragères par les éleveurs. En dépendent à la fois les quantités, qualité et disponibilité des fourrages dans le temps. Ceux-ci sont 


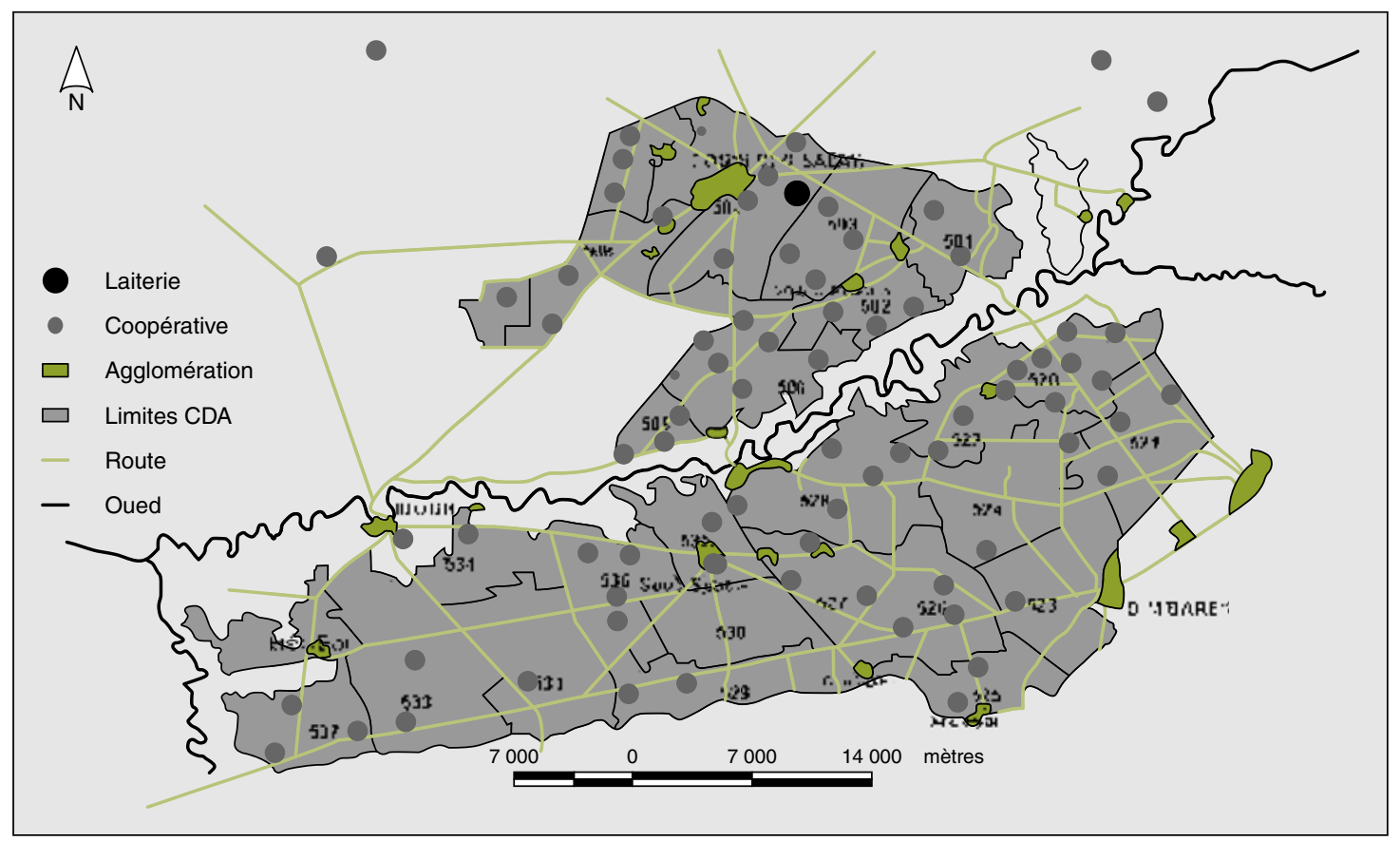

Figure 1. Localisation des coopératives de collecte et de la laiterie sur le périmètre du Tadla (source Ormvat).

Figure 1. Location of the dairy plant and the collecting co-operatives in the Tadla irrigated scheme (Ormvat source).

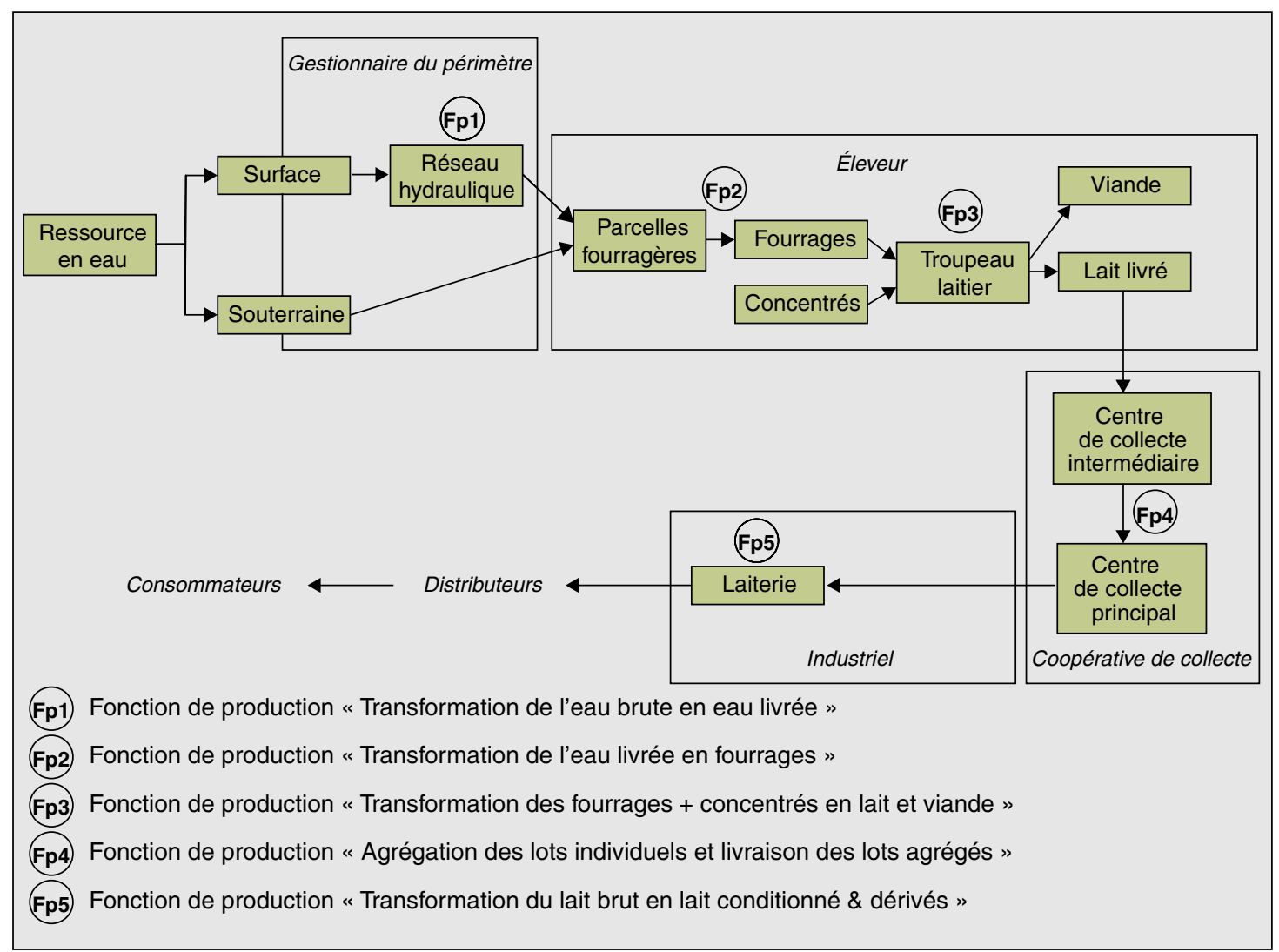

Figure 2. Représentation schématique de la chaîne d'approvisionnement laitière dans le périmètre irrigué du Tadla.

Figure 2. Schematic overview of the dairy supply chain in the Tadla irrigation scheme. 
ensuite associés par l'éleveur à d'éventuels concentrés pour constituer le système alimentaire des animaux. Ces rations sont transformées en lait et viande via une troisième fonction de production $F_{p}^{3}$ faisant appel aux mécanismes physiologiques propres aux races et individus présents dans le troupeau. Les éleveurs commercialisent une partie du lait à la laiterie via les centres de collecte gérés par les coopératives. Ces coopératives agrègent les livraisons individuelles en volumes plus importants, refroidis et transportés vers l'usine par camionciterne (fonction de production $F_{p}{ }^{4}$ ). La laiterie transforme le lait brut en lait conditionné et en dérivés (fonction de production $F_{p}{ }^{5}$ ) qui sont alors distribués aux consommateurs selon différents canaux (grande distribution, détaillants). À ces différents maillons sont associés des flux d'information circulant vers et à partir des coopératives de collecte. Les volumes livrés par les éleveurs sont en effet mesurés à l'entrée du centre de collecte, alors que les volumes agrégés provenant des coopératives sont mesurés à l'entrée de la laiterie. Ces mesures, auxquelles s'ajoutent des contrôles de qualité à l'usine, fournissent la base du paiement du lait par l'industriel vers la coopérative, puis de la coopérative vers les éleveurs.

\section{Intérêts d'un dispositif de coordination}

Le gestionnaire du périmètre et l'industriel ont des objectifs spécifiques qu'ils souhaitent voir partager par les agriculteurs. Le premier vise à économiser l'eau, devenue ressource rare, et à améliorer sa valorisation économique tout en couvrant ses coûts de service, ce qui renvoie explicitement aux types de production présents sur le périmètre. Ainsi, la luzerne est une culture très consommatrice en eau, de l'ordre de $15000 \mathrm{~m}^{3} /$ ha avec des variations de 1 à 4 selon les agriculteurs (Kuper et al., 2003). La valorisation de l'eau par l'élevage, lait et viande combinés, est difficile à évaluer, mais peut être améliorée par de meilleures performances de la chaîne d'approvisionnement en lait (Kuper et al., 2005). Cette amélioration dépend des trois objectifs stratégiques que se fixe l'industriel : i) augmenter la production laitière totale en améliorant la productivité par vache qui, avec 3000 litres par an, est faible au vu du

\section{Tableau 1. Présentation de la typologie des exploitations laitières sur le périmètre irrigué du Tadla.}

Table 1. Presentation of the dairy farm typology in the Tadla irrigation scheme.

\begin{tabular}{|c|c|}
\hline $\begin{array}{l}\text { Type 1. Investissement de capitaux } \\
\text { dans une étable laitière } \\
\text { - Troupeau de } 20 \text { VL ou plus } \\
\text { - Système fourrager diversifié et non } \\
\text { limité par la surface disponible } \\
\text { - Livraison directe à l'usine } \\
\text { - Employés salariés } \\
\text { - } 3000 \text { à } 5000 \text { L/NL/an }\end{array}$ & $\begin{array}{l}\text { Type 3. Élevage bovin en développement } \\
\text { dans une exploitation diversifiée } \\
\text { - } 10 \text { à } 20 \text { hectares de surface disponible } \\
\text { - } 3 \text { à } 6 \text { hectares de surface fourragère } \\
\text { - Investissements dans des bâtiments et } \\
\text { des équipements de traite } \\
\text { - } 8 \text { VL et projet d'augmentation } \\
\text { - } 2500 \text { à } 3000 \mathrm{~L} / \mathrm{NL} / \mathrm{an}\end{array}$ \\
\hline $\begin{array}{l}\text { Type 2. Élevage bovin valorisant } \\
\text { un foncier limité } \\
\text { - Surface disponible de } 2 \text { hectares } \\
\text { entièrement en cultures fourragères } \\
\text { - Investissement dans des bâtiments } \\
\text { et des équipements de traite } \\
\text { - } 5 \text { à } 10 \text { VL } \\
\text { - } 2500 \text { à } 5000 \mathrm{LN} \text { L/an }\end{array}$ & $\begin{array}{l}\text { Type 4. Élevage bovin au service } \\
\text { d'un système de polyculture } \\
\text { - } 7 \text { hectares de surface disponible, dont } \\
15 \% \text { en cultures fourragères } \\
\text { - Race locale adaptée aux restrictions } \\
\text { alimentaires } \\
\text { - Recyclage des sous-produits végétaux } \\
\text { - Le lait assure la trésorerie } \\
\text { - } 1500 \text { à } 2000 \text { L/NL/an }\end{array}$ \\
\hline
\end{tabular}

VL : vache laitière.

potentiel génétique exploité (races Montbéliarde ou Holstein); ii) réduire la saisonnalité des apports de 1,9 à 1,6 entre périodes de haute lactation, du 15 février au 14 août, et basse lactation, du 15 août au 14 février ; iii) améliorer la qualité du lait livré, tant sur le plan hygiénique que chimique.

La réalisation de ces objectifs dépend largement des agriculteurs, dont l'atomisation et la diversité de la demande en eau ou de l'offre en lait sont mal appréhendées par le gestionnaire du périmètre comme par l'industriel. Partant d'une enquête sur 32 éleveurs, quatre types ont été identifiés à partir de leurs stratégies, leurs potentialités et leurs problèmes spécifiques vis-à-vis de la production laitière (tableau 1). Ainsi, le type 2 présente une orientation laitière marquée, mais sa pro-

\section{Tableau 2. Résultats économiques de l'atelier bovin des quatre types d'exploitations (source : Kuper et al., 2006).}

Table 2. Economic results of the cattle production in the four types of farm (source: Kuper et al., 2006).

\begin{tabular}{|c|c|c|c|c|}
\hline & Type 1 & Type 2 & Type 3 & Type 4 \\
\hline Total lait livré (I/an) & 324000 & 15863 & 11296 & 2241 \\
\hline $\begin{array}{l}\text { Valeur ajoutée brute (VAB) par vache } \\
\text { (en dinars) }\end{array}$ & 13666 & 3370 & 1455 & 3442 \\
\hline $\begin{array}{l}\text { Produit viande/produit total atelier } \\
\text { élevage (\%) }\end{array}$ & 21 & 39 & 48 & 71 \\
\hline $\begin{array}{l}\text { Produit lait/produit total atelier } \\
\text { élevage }(\%)\end{array}$ & 64 & 61 & 41 & 29 \\
\hline VAB bovin/NAB totale exploitation (\%) & 72 & 100 & 9 & 6 \\
\hline
\end{tabular}

11 dinars $=1$ euro. duction fourragère et la taille de son troupeau sont contraintes par son foncier limité. Ses performances sont très hétérogènes, laissant supposer des problèmes de maîtrise du rationnement des animaux. À l'opposé, le type 4 présente peu d'intérêt pour l'industriel dans la mesure où l'élevage n'y est qu'une activité de complément basée sur un troupeau de race locale ou d'animaux de type croisé faiblement productifs. La part de l'activité laitière et de la production de viande dans la valeur ajoutée brute dégagée par chaque type varie fortement en fonction de ces orientations stratégiques (tableau 2). Dans ce contexte, clients et fournisseurs sont étroitement liés. Le gestionnaire de périmètre et l'industriel disposent d'un quasi-monopole, respectivement de fourniture du service de l'eau et d'achat du 
lait. Mais le maintien de leur activité suppose que les agriculteurs puissent payer le service de l'eau et fournir un produit conforme au cahier des charges de la laiterie. Cette interdépendance est accrue par l'existence d'actifs industriels et hydrauliques spécifiques et par des contraintes logistiques liées à l'atomisation des agriculteurs et à au caractère périssable du lait.

Dans ce contexte, les échanges entre acteurs ne peuvent être régulés par une coordination purement marchande. Des dispositifs spécifiques sont nécessaires pour réduire les asymétries d'information et minimiser les risques d'opportunisme (Holmstrom et Milgrom, 1994). Ils se fondent ici sur trois grandes composantes : une organisation des flux de matière première et d'eau, des outils économiques et des services liés aux fonctions centrales de fournisseur d'eau et de collecteur de lait.

\section{L'organisation des flux d'eau et de lait}

L'organisation des flux consiste à planifier et piloter dans le temps et l'espace la distribution de l'eau et les livraisons de lait. L'Ormvat se fonde sur un schéma en deux temps. Les besoins en eau annuels du périmètre sont d'abord estimés à partir des assolements annoncés par les producteurs. Chaque culture est affectée d'une surface et d'un nombre d'irrigations par saison. Des règles de priorité sont utilisées face aux limites du système d'irrigation et de la ressource en eau :

1. Assurer les besoins minimaux des cultures pérennes, dont la luzerne ;

2. Privilégier la betterave sucrière, afin de sécuriser l'approvisionnement des sucreries ;

3. Assurer les besoins des superficies céréalières.

L'eau est ensuite distribuée sur la base d'un tour d'eau hebdomadaire. Les agriculteurs s'ajustent à cette offre en réallouant leur dotation selon leurs objectifs propres et en complétant les apports de l'Ormvat par l'eau pompée dans la nappe s'ils disposent d'un forage.

La gestion des flux de lait est organisée en plusieurs étapes décrites à la figure 3 (Kuper et al., 2006). La multiplicité des livraisons individuelles sur de faibles volumes journaliers (quelques litres à quelques dizaines de litres) nécessite une première agrégation des lots au niveau des tanks des centres de collecte. Les mesures de qualité sur les lots individuels étant réduites au mouillage et à l'acidité pour des raisons pratiques et économiques, la traçabilité ne peut être assurée jusqu'aux élevages et les incitations individuelles visant à améliorer la qualité du lait demeurent limitées. Cet objectif passe par des actions plus structurelles, telles que rapprocher les centres de collecte des livreurs ou diffuser l'utilisation des bidons en aluminium. Elles ne concernent que la qualité hygiénique du lait, alors que l'industriel affiche également des objectifs d'amélioration de sa qualité chimique.

La coordination entre flux d'eau gérés par l'Ormvat et flux de lait gérés par la laiterie ne fait appel à aucun dispositif formel. Les éleveurs, qui contrôlent la transformation de l'eau en lait (figure 2) répartissent la première entre différentes productions et utilisent les ressources fourragères à différentes fins (lait, viande, vente de foin). La diversité de comportements des 17000 éleveurs complique les possibilités de coordination entre le périmètre et le bassin de collecte laitier. Or, sans coordination, une forte demande en eau des agriculteurs, stimulée par la demande en lait de la laiterie, pourrait ne

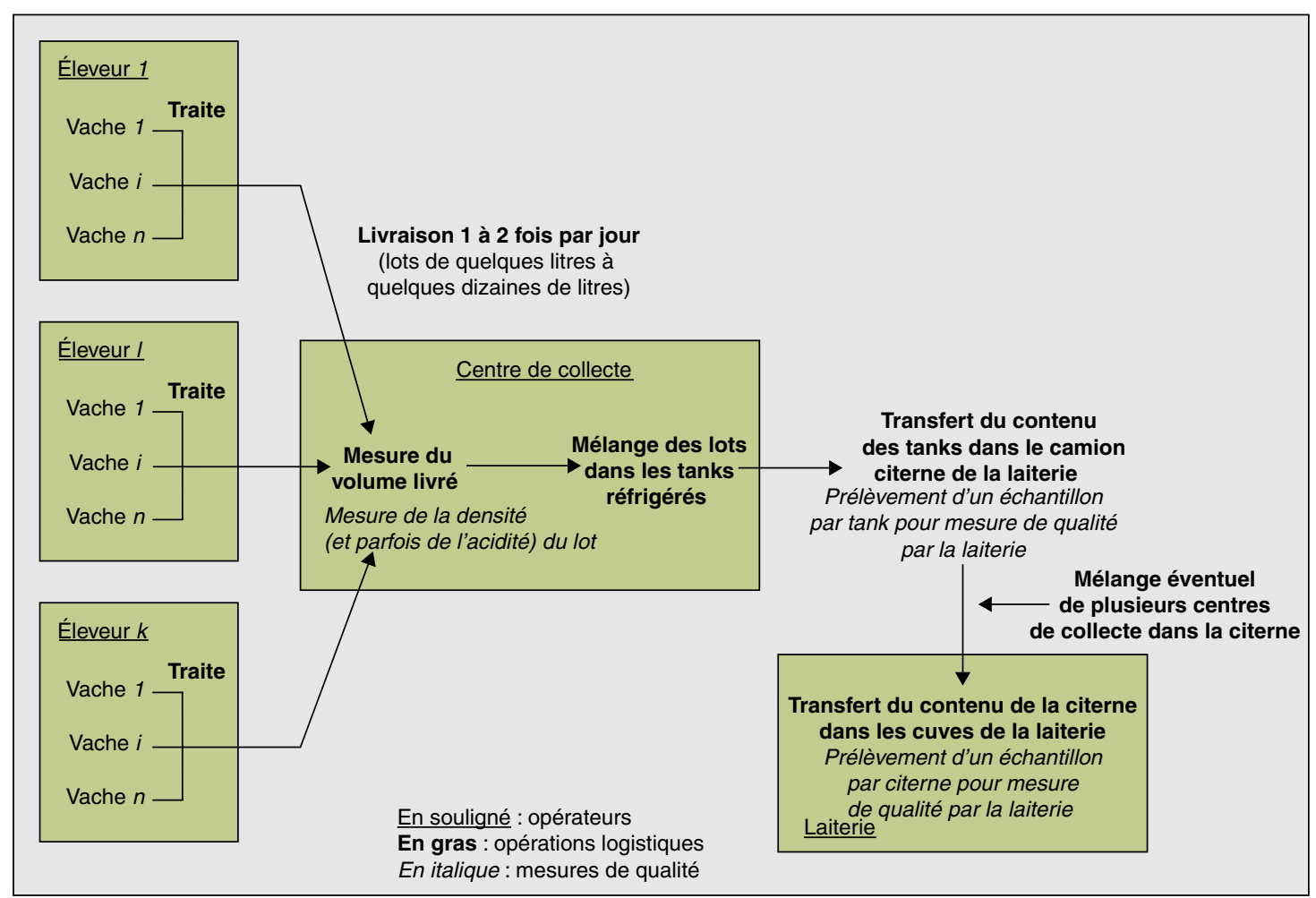

Figure 3. Organisation logistique des flux de lait des éleveurs à la laiterie (source: Kuper et al., 2006).

Figure 3. Logistics of milk flows from dairy farms to the dairy plant (source: Kuper et al., 2006). 
pas être couverte par le gestionnaire du périmètre. Celui-ci est en effet confronté à des arbitrages entre la ressource allouée par l'agence de bassin et les demandes des différentes chaînes d'approvisionnement potentiellement concurrentes. Les outils économiques mis en place peuventils aider à résoudre ces arbitrages?

\section{Outils économiques et services liés}

Les outils économiques utilisés par le gestionnaire de périmètre (tarification de l'eau) et par l'industriel (système de paiement du lait) visent à couvrir leurs coûts, tout en incitant les agriculteurs à adopter des comportements conformes à leurs objectifs. Ainsi, la tarification de l'eau dans un contexte de ressource rare cherche à favoriser son allocation aux cultures qui la valorisent le mieux (Le Gal et al., 2003). Elle suppose que les agriculteurs adoptent un comportement économique rationnel en réponse au signal transmis par le prix qu'ils doivent acquitter. Le système tarifaire adopté dans le Tadla est de type proportionnel, basé sur les volumes consommés à l'entrée de l'exploitation (Hellegers et al., 2007). Le montant appliqué par $\mathrm{m}^{3}$ permet de couvrir les coûts complets d'opération et de maintenance tout en demeurant le plus faible du Maroc, du fait de l'absence de coûts énergétiques de relevage (irrigation entièrement de type gravitaire). Ce système a l'avantage d'établir une relation directe entre le volume consommé par l'agriculteur et sa facture, le rendant a priori plus sensible aux économies d'eau. Mais il a le défaut d'être affecté par les conditions climatiques, les recettes du gestionnaire diminuant en année sèche du fait d'une offre en eau réduite, et en année humide lorsque la demande faiblit.

Le système de rémunération du lait cherche à augmenter et réguler les productions livrées, tout en améliorant leur qualité. Le lait est payé au litre, selon un prix de base en haute lactation augmenté de $15 \%$ en basse lactation. Des primes sont payées aux coopératives en fonction de leurs caractéristiques (nombre de collectes par jour et saisonnalité de leurs approvisionnements) et de leurs taux moyens en matière grasse. Ces primes ne sont pas redistribuées individuellement aux éleveurs faute d'analyse des lots individuels. Elles vont nourrir les excédents financiers des coopératives, qui sont réinvestis dans des activités de service à leurs membres (Oudin, 2006).

L'efficacité de ce système de rémunération est cependant limitée. Des simulations ont montré que l'écart de prix entre basse et haute lactation semble insuffisant pour compenser les efforts que les éleveurs devraient fournir pour alimenter les vaches en production en hiver et synchroniser leurs vêlages sur de faibles effectifs (Kuper et al., 2006). Le paiement à la qualité bute sur l'agrégation des lots dans les centres de collecte et l'impossibilité d'établir une relation directe entre la laiterie et l'éleveur.

Pour pallier ces insuffisances, l'Ormvat et la laiterie collaborent pour offrir aux éleveurs des services complémentaires à leur fonction principale. Outre des formations, des événements (foire agricole, séances d'information), un suivi et un encadrement technique de certains élevages, des crédits d'équipement (machines à traire, pots et tanks en aluminium) sont mis à disposition des centres de collecte et des exploitations individuelles. Combinée à l'organisation de la distribution de concentrés, la politique de crédit vise également à faciliter l'achat de génisses améliorées. Enfin, l'insémination artificielle est pratiquée à grande échelle, avec la mise à disposition d'inséminateurs et de semence amélioratrice. Ces services sont également proposés par l'association des éleveurs du Tadla (AET) à ses coopératives adhérentes, en complément de son rôle de défense des intérêts des éleveurs.

Ce dispositif de coordination depuis la fourniture d'eau jusqu'à l'approvisionnement de la laiterie présente in fine des résultats mitigés. La productivité par vache demeure perfectible au regard du potentiel génétique des troupeaux, la saisonnalité des livraisons est encore trop marquée, et la qualité bactériologique souffre du manque d'hygiène dans les étables et de ruptures dans la chaîne du froid. De plus, la luzerne consomme beaucoup d'eau, dont la valorisation économique est difficile à évaluer.

\section{Conclusions}

Cette analyse souligne l'intérêt d'intégrer, dans une même chaîne d'approvisionnement, le périmètre irrigué fournisseur d'eau, les agriculteurs producteurs de matière première agricole et les entreprises de transformation et distribution. Au plan théorique, ce cas permet de traiter des questions peu abordées dans la littérature, telles que la relation entre organisation des flux et système de rémunération (Le Gal et al., 2006) ou la conception de systèmes d'incitation adaptés à des relations indirectes entre fournisseurs et clients.

Au plan opérationnel, l'analyse des modes de coordination entre fonctions et acteurs permet d'identifier les actions à même d'améliorer les performances de l'ensemble tels que : i) la mise en place d'une organisation efficace des flux d'eau et de matière première ; ii) la conception de systèmes de tarification et de rémunération adaptés aux objectifs recherchés; et iii) la prise en compte des relations croisées entre ces deux leviers d'intervention. Ainsi, le mode de paiement à la qualité du lait est lié à l'organisation logistique de la collecte et à la difficulté de réaliser des mesures sur les faibles volumes livrés individuellement. Dans ce contexte, les agriculteurs et les coopératives doivent faire l'objet d'une attention particulière, compte tenu de leur poids dans la détermination de la quantité, la qualité et la temporalité de la production et de leur diversité à l'échelle du bassin de collecte et du périmètre.

Sur cette base, différents axes de recherche-intervention ont été discutés et validés par les acteurs. La mise au point d'une démarche d'aide à la conception de systèmes d'alimentation des vaches laitières sous contraintes de ressources en eau, en terre et en trésorerie, est apparue prioritaire au vu des tendances à substituer la luzerne par le maïs ensilage, supposé moins consommateur en eau. Ces deux fourrages différant en termes d'apport d'azote et d'énergie, il convient de revoir les rations dans une perspective de diversification des systèmes fourragers.

La gestion de la qualité du lait depuis l'étable jusqu'à la laiterie est également un sujet stratégique, dont dépend pour partie la valeur créée par le bassin d'approvisionnement. Il renvoie au rôle des coopératives de collecte, dont l'amélioration des modalités de gestion et des performances est un thème de recherche et d'intervention en soi, associé à la conception d'un système de rémunération prenant mieux en compte les efforts individuels. 


\section{Références}

Beamon B. Supply chain design and analysis : models and methods. Int J Prod Econ 1998 $55: 281-94$.

Chen IJ, Paulraj A. Towards a theory of supply chain management : the construct and measurements. J Oper Manage 2004 ; 22 : 119-50.

Corniaux C, Duteurtre G, Dieye PN, Poccard Chapuis R. 2005. Les minilaiteries comme modèle $d^{\prime}$ 'organisation des filières laitières en Afrique de l'Ouest : succès et limites. Rev Elev Med Vet Trop $2005 ; 58: 237-43$.

Croom S, Romano P, Giannakis M. Supply chain management: an analytical framework for critical review. European Journal of Pur chasing \& Supply Management 2000; 6 67-83.

Gaucher S, Le Gal PY, Soler LG. Modelling supply chain management in the sugar industry. Sugar Cane International 2004 ; 22 : 8-16.

Giard V. Gestion de la production. Paris : Economica, 1988.

Hellegers PJGJ, Perry CJ, Petitguyot T. Water Pricing in Tadla, Morocco. In: Molle F Berkoff J, Barker R, eds. Irrigation water pricing policy in context: exploring the gap between theory and practice. Wallingford (United Kingdom) : International Water Management Institute (IWMI); CABI publishing 2007 (forthcoming).
Holmstrom B, Milgrom P. The firm as an incentive system. Am Econ Rev 1994 ; 84 : 972-91.

Kuper M, Hammani A, Zemzam S, Bouarfa S, Thomas $V$. Stratégies d'utilisation conjuguée des eaux de surface et souterraine pour l'irrigation : le cas du périmètre irrigué du Tadla au Maroc. In : Le Goulven P, Bouarfa S, Kuper M, eds. Proceedings of the 3rd Pcsi seminar on the Integrated water management in river basins. Montpellier ; Paris : Centre de cooperation internationale en recherche agronomique pour le développement (Cirad); Institut de recherche pour l'ingénierie de l'agriculture et de l'environnement (Cemagref): Institut de recherche pour le développement (IRD), 2003.

Kuper M, Le Gal PY, Sraïri MT, Moulin $\mathrm{CH}$ Puillet L. Increasing irrigation water productivity through supply chain management of agro food products : the case of dairy farming in the Tadla irrigation scheme (Morocco). In : Instruments économiques et modernisation de l'agriculture irriguée en méditerranée. Séminaire Wademed Kairouan. 2005.

Kuper M, Le Gal PY, Moulin CH, Puillet L, Sraïri MT, Elbahri M. Typologie et modélisation des exploitations laitières sur le périmètre irrigué du Tadla (Maroc). Montpellier : Centre de coopération internationale en recherche agronomique pour le développement (Cirad), Département Territoires, ressources, acteurs (Cirad-Tera), 2006
Le Bail M. Le bassin d'approvisionnement: territoire de la gestion agronomique de la qualité des productions végétales. In : Prevost $P$, ed. Agronomes et territoires. Paris : L'Harmat$\tan , 2005$.

Le Gal PY, Rieu T, Fall C. Water pricing and sustainability of self-governing irrigation schemes. Irrigation and Drainage Systems $2003 ; 17: 213-38$

Le Gal PY, Papaïconomou H, Meyer E, Lyne P. Combined impact of alternative relative cane payment systems and harvest scheduling on growers' revenues. Sugar Cane International $2006 ; 24: 14-20$.

Oudin E. Analyse et propositions d'améliorations du fonctionnement de coopératives de collecte laitière au Maroc: approche par les systèmes d'information. Paris: Institut national agronomique de Paris-Grignon (Ina-Pg); Centre de cooperation internationale en recherche agronomique pour le développement (Cirad), 2006

Shah N. Process industry supply chains advances and challenges. Comput Chem Eng $2005 ; 29$ : 1225-35.

Sraïri MT, El Khattabi M. Évaluation économique et technique de la production laitière intensive en zone semi-aride au Maroc. Cah Agric $2001 ; 10: 51-5$ 